\title{
Oral fluorescein angiography: reassessment of its relative safety and evaluation of optimum conditions with use of capsules
}

\author{
A P Watson, E S Rosen
}

\begin{abstract}
Injection of fluorescein intravenously for fundal angiography is associated with a high incidence of minor adverse effects (21\%) but a very low incidence of serious (life threatening) reactions $(0 \cdot 05 \%)$. A serious reaction may occur without warning in a patient with no history of atopy. There are no reports of oral fluorescein causing a serious reaction, and minor adverse effects are uncommon. A study was undertaken to determine optimum conditions for oral fluorescein angiography. Capsules proved more convenient than a solution for ingestion of fluorescein. A dose of $25 \mathrm{mg} / \mathrm{kg}$ body weight produced good quality angiograms in $75 \%$ of the patients. The best pictures were obtained by photographing the fundus after 40 and 60 minutes.
\end{abstract}

Fluorescein fundal angiography (FFA) has become progressively established as a valuable clinical investigation since the technique was described in detail in 1961.' When fluorescein is given intravenously reactions can occur. Giving the fluorescein orally makes the risk of a serious reaction minimal and greatly reduces the incidence of minor reactions. Previous authors have already reported that good quality angiograms can be produced with oral fluorescein, ${ }^{2-6}$ though high intensity early passes do not occur.

The aims of this study were to evaluate the use of oral fluorescein in capsule form, to determine if there is a dose which will reliably give good quality angiograms, and to discover the best time to photograph the fundus after the capsules have been taken.

\section{Materials and methods}

After the approval of the ethical committee 20 healthy volunteers were recruited from the staff of Manchester Royal Eye Hospital and were asked to give written consent. The subjects were asked to fast for six hours before being given a test dose of $10 \mathrm{mg}$ of fluorescein in liquid form and being weighed. If over the next half hour they experienced no symptoms such as faintness, warmth, or itching suggestive of an allergic reaction, they were then given a larger dose in capsule form. ${ }^{\star}$ The patients were randomly assigned to one of five groups, to be given $1,1 \cdot 5$, *Capsules of sodium fluorescein $500 \mathrm{mg}$ were formulated and prepared by Jill Bumphrey, MPS, at the Leicestershire Health Authority District Pharmacy, Leicester Royal Infirmary, Leicester. The raw material specification was fluorescein sodium BP, NWRHA specification, supplied by Société Chimique Pointet Gorard. The pharmacy can supply the capsules at a cost of approximately $£ 10$ per 100 .
2 , or $2 \cdot 5 \mathrm{~g}$ of fluorescein. One pupil was dilated with tropicamide $1 \%$, plus phenylephrine $10 \%$ if necessary.

Fundal photographs were taken with either a Canon CF-60U or Kowa Pro I fundus camera 20, 30, 40 and 60 minutes after the capsules had been swallowed. The film used was Ilford FP4 rated to 800 ASA. The films were developed in Kodak D76 for 30 minutes at $20^{\circ} \mathrm{C}$.

\section{Results}

The 80 angiograms were graded into four categories: $3=$ strong fluorescence; $2=$ moderate fluorescence; $1=$ faint fluorescence; $0=$ no useful fluorescence.

Grade 3 angiograms were comparable in quality with those produced by intravenous fluorescein. Grade 2 angiograms were also of good quality, and any abnormal leakage would be readily seen. In the grade 1 angiograms the disc and macula could be distinguished and it would be possible to recognise any major leakage. However, small amounts of late leakage might be missed. Grade 0 angiograms did not show any significant degree of fluorescence, and fundal features could not be distinguished. Examples of angiograms from categories 1,2 , and 3 are shown in Figure 1.

For 15 of the 20 volunteers one or more angiograms in grade 2 or 3 were produced, and 12 of these 15 had one or more grade 3 angiograms. All except one had been given a dose of fluorescein equivalent to at least $25 \mathrm{mg} / \mathrm{kg}$ body weight. The best quality angiograms were obtained at 40 or 60 minutes after they had taken the capsules, though good quality angiograms could also be obtained at 30 minutes in some cases. An example of progression of fluorescence in successive angiograms is shown in Figure 2.

For five of the 20 subjects no useful angiograms were obtained. In four of these five there was no visible fluorescence. Although two of the five had been given fluorescein equivalent to less than $15 \mathrm{mg} / \mathrm{kg}$ body weight, the other three had received a dose of over $30 \mathrm{mg} / \mathrm{kg}$.

None of the subjects experienced any reaction to the test dose of liquid fluorescein. After taking the capsules one person developed a mild urticarial rash on the extensor surfaces of her arms and legs. She experienced no other ill effects. She was given chlorpheniramine maleate $4 \mathrm{mg}$ by mouth, and the rash resolved over the next four hours.

\section{Discussion}

Fluorescein was first synthesised in 1871 . It was 


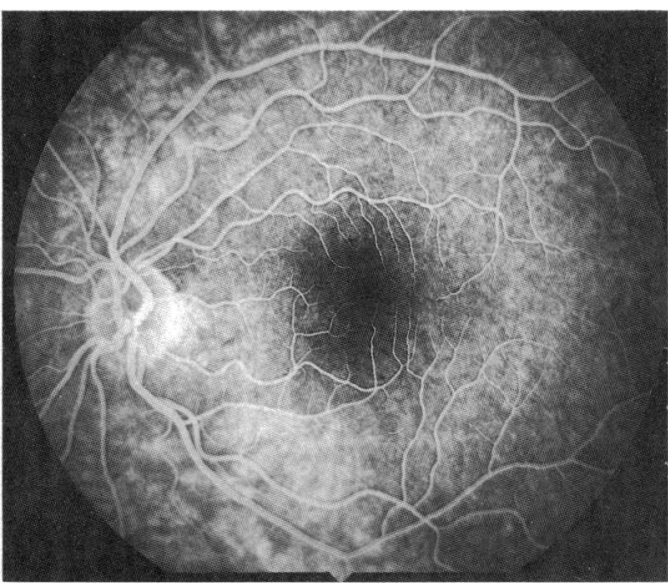

Figure 1A: Grade 3

Figure 1: Examples of different grades of angiogram.

used in the examination of the fundus in Germany in $1910^{7}$ and in a study of a patient in the USA with abnormal aqueous dynamics in the SturgeWeber syndrome in $1932 .{ }^{8}$ By 1943 its use was recommended as an adjunct to ocular examination in Duke-Elder's Textbook of ophthalmology published in the UK and USA. ${ }^{9}$

In modern ophthalmic practice fluorescein fundal angiography (FFA) can provide valuable information about the retinal circulation, the integrity of the blood-retinal barrier, and the health of the retinal pigment epithelium. ${ }^{10}$ Intravenous fluorescein angiograms are of a reliably high quality if the ocular media are clear. The fluorescein is usually given intravenously, and, as with almost any agent given by this route, there is a risk of provoking a reaction. Most reactions to intravenous (IV) fluorescein are mild, but a small proportion are serious and occasionally fatal.

Mild reactions include nausea, vomiting, chills or warmth, hypersalivation, dizziness, urticaria, itching, and facial oedema. The following serious reactions have been reported: wheezing, laryngeal oedema, bronchospasm, pulmonary oedema, respiratory arrest, basilar artery ischaemia, atrial fibrillation, chest pain, myocardial infarction, loss of consciousness, and shock. ${ }^{11-16}$ Skin necrosis may occur if there is extravasation of the fluorescein. ${ }^{17}$

The incidence of reactions is not easy to establish from the literature. In a prospective study where reactions to IV fluorescein were

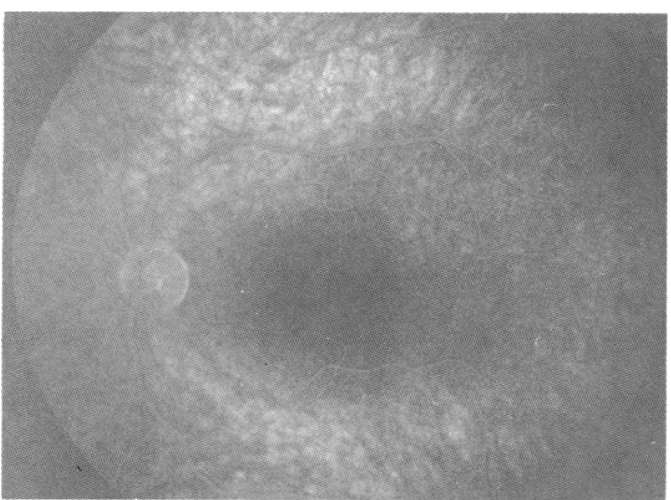

Figure 1C: Grade 1.

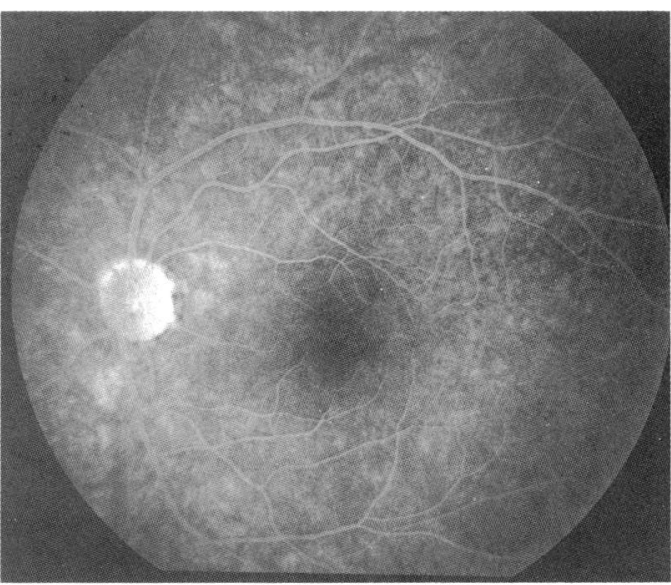

Figure 1B: Grade 2.

ascertained by asking each patient to describe any unpleasant sensations, as well as by observation, an incidence of $21 \%$ was reported, ${ }^{18}$ though all these were mild. In a retrospective study reviewing the records of a number of different institutions in the USA another study found the incidence of reactions to be $0.6 \%$, but of these the majority $(66.6 \%)$ were severe. ${ }^{11}$ Figures from other studies lie between the two extremes, suggesting that mild reactions are common, occurring in about $20 \%$ of injected patients, but that life threatening reactions are rare, occurring in less than $0.05 \%$ of patients. This risk factor is nevertheless significant for a procedure which is most commonly undertaken in situations where life is not threatened by the condition being investigated.

There is greater likelihood of a reaction to IV fluorescein in a patient with a history of atopy or drug reactions, "but a life threatening reaction, such as acute pulmonary oedema, may occur in a patient who is not atopic and has no respiratory disease. ${ }^{12}$

The precise nature of reactions to IV fluorescein is not clear. Some may be due to hypersensitivity (anaphylaxis) as a result of an immunological response of the host to a hapten (drug)-protein combination. Yet the proportion occurring in patients who have received previous injections is not much greater than in patients exposed to fluorescein (in large amounts) for the first time. It is hard to imagine how people who have not been previously injected could become sensitised to fluorescein either through topical applications to their eyes or from the environment. Skin testing with both fluorescein and fluorescein-conjugated human serum albumin (F-HSA) in three patients who had suffered reactions to IV fluorescein yielded only one positive result, and that was to F-HSA."

Raised serum histamine levels have been found after the administration of IV fluorescein to people who have experienced a reaction, but also in a large proportion of those who experienced no ill effects. ${ }^{18}$ In spite of the finding of raised levels in unaffected patients, these results do suggest that histamine plays a part in the production of reactions to IV fluorescein. Apart from its role in anaphylaxis, histamine may also be released (in vitro) in the absence of an antigenantibody reaction. ${ }^{19}$ 


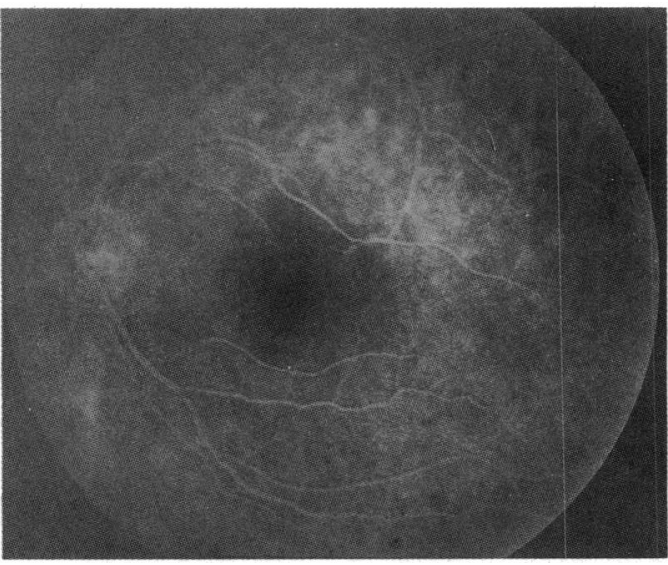

Figure 2A: 20 minutes.

Figure 2: Successive angiograms in an individual.

It may also have been of importance that in the past the solutions of fluorescein used for FFA contained significant quantities of contaminants. Metallic and ionic mercury were found in samples in the USA in 1970, ${ }^{11}$ and after a number of serious reactions in the UK high levels (up to $2 \%$ ) of dimethyl formamide were isolated. ${ }^{20} \mathrm{At}$ that time dimethylformamide was difficult to eliminate entirely during the manufacture of fluorescein, and an upper limit specification of $0.2 \%$ was set by the Department of Health and Social Security. ${ }^{21}$

A serious reaction to any potentially allergenic agent is much less likely if it is administered orally rather than systemically. As described in the introduction, fluorescein has been administered orally for many years and has recently been used by an increasing number of investigators. To the best of our knowledge no serious reaction to oral fluorescein has ever been reported. There is only one previously reported 'mild allergic reaction', ${ }^{3}$ and one of our volunteers developed a mild, transient, urticarial rash.

Oral fluorescein produces good quality angiograms in most cases provided it is not necessary to have early phase, high intensity pictures. Angiograms from oral fluorescein have already been shown to be useful in the demonstration of cystoid macular oedema, ${ }^{23622}$ papilloedema, ${ }^{19224}$ and central serous retinopathy. ${ }^{5}$ The advantages of oral fluorescein are that it need not be given by

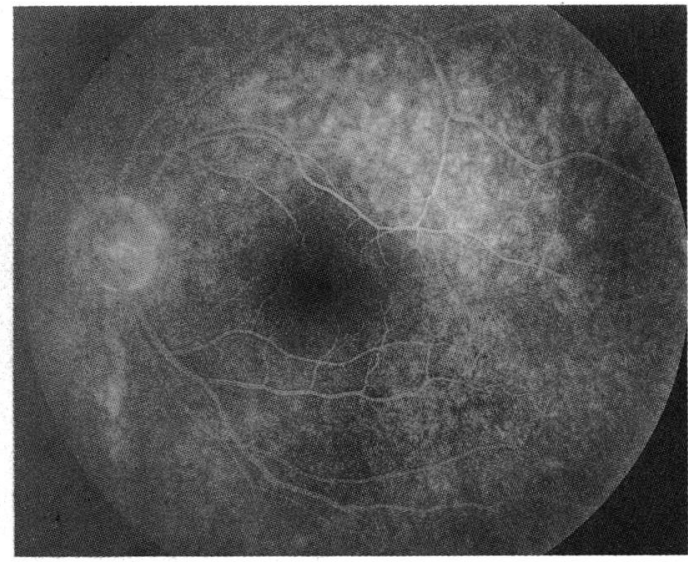

Figure 2B: 30 minutes.

a doctor, patients with inaccessible veins can be investigated, children or adults who are afraid of injections are not alarmed, and it is safer than intravenous fluorescein.

As fluorescein capsules have not previously been available, it has been customary to give oral fluorescein in liquid form, from phials manufactured for intravenous use. The disadvantages of liquid fluorescein are that it looks and tastes unpleasant (even when mixed with fruit juice or cordial), that it temporarily stains teeth, and that, if there is any spillage on to clothes, the results are spectacular. The use of capsules avoids all these problems.

Our results indicate that a dose of $25 \mathrm{mg} / \mathrm{kg}$ body weight produces good quality angiograms in $75 \%$ of patients. In order to provide comparable results the volunteers for this study were asked to fast before taking the fluorescein, but from earlier experience the authors have found that this is not essential, and for patients with diabetes mellitus it is inadvisable to recommend fasting before oral fluorescein angiography.

In this study the best pictures were obtained by photographing the fundus at 40 and 60 minutes after the capsules had been taken. We recommend the use of oral fluorescein in capsule form when early, high intensity, angiograms are not required, on the grounds of efficiency, convenience, and safety.

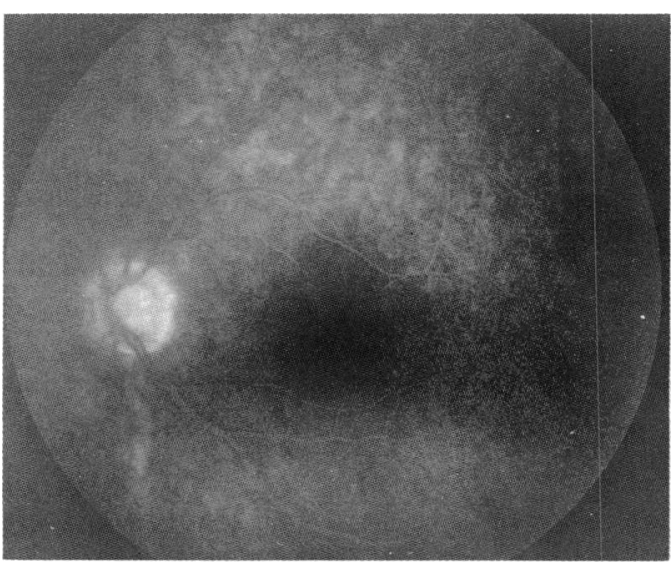

Figure 2C: 40 minutes.

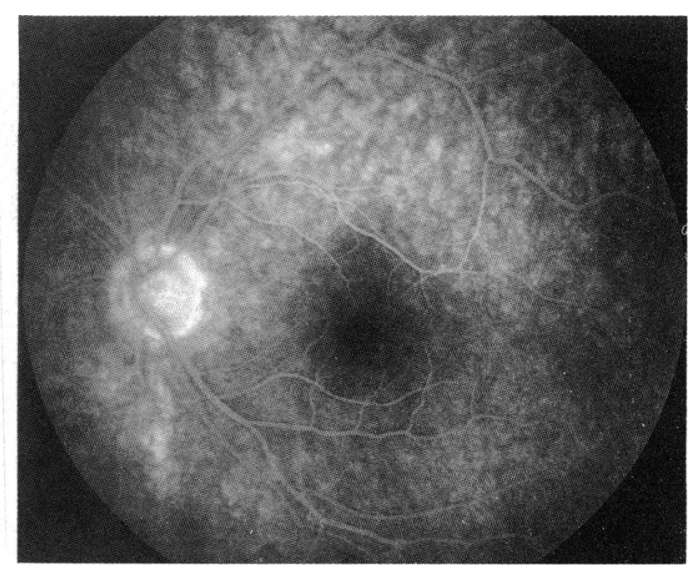

Figure 2D: 60 minutes. 
All the photographs were taken by Richard Hancock (general medical photographer) and Adam Prest (medical research photographer), whose skill and commitment enabled this study to come to a successful conclusion.

The authors wish to thank those friends and colleagues at Manchester Royal Eye Hospital who volunteered to take oral fluorescein for this study, which would not have been possible without their involvement.

1 Novotny HR, Alvis DL. A method of photographing fluorescence in circulating blood in the human retina. Circulation 1961; 24: 82-6.

2 Kelley JS, Kincaid M. Retinal fluorography using ora fluorescein. Arch Ophthalmol 1979; 97: 2331-2.

3 Kelley JS, Kincaid M, Hoover RE, McBeth C. Retinal fluorograms using oral fluorescein. Ophthalmology 1980; 87: 805-11.

4 Oral fluorescein study group. Oral fluorography. $7 \mathrm{Am} \mathrm{Optom}$ Assoc 1985; 56: 784-92.

5 Azad R, Nayak BK, Tewari HK, Khosola PK. Oral fluorescein angiography. Indian $\mathcal{F}$ Ophthalmol 1984; 32:

6 Noble MJ, Cheng H, Jacobs PJ. Oral fluorescein and cystoid macular oedema: detection in aphakic and pseudophakic eyes. Br f Ophthalmol 1984; 68: 221-4.

7 Burk A. Die klinische, physiologische und pathologische Bedeutung der Fluoreazenz in augenach Darreichung von Uranin. Klin Monatsbl Augenheilkd 1910; 48: 445-54.

8 Tyson HH. Nevus flammeus of the face and globe associated with glaucoma, vascular changes in the iris and a calcified vascular growth in the occipital lobe of the brain, with right vascular growth in the occipital lobe of the brain, with right
homonymous hemianopia. Arch Ophthalmol 1932; 8: 36571 .

9 Duke-Elder S. Textbook of ophthalmology. London: Kimpton, 1943: 2: 1150

10 Rosen ES. Fluorescence photography of the eye. London Butterworths, 1969.

11 Stein MR, Parker CW. Reactions following intravenous fluorescein. Am f Ophthalmol 1971; 72: 861-8.
12 Hess JB. Pacuraiu RJ. Acute pulmonary edema following intravenous fluorescein angiography. $\mathrm{Am} \mathcal{f}$ Ophthalmol $1976 ; 82: 567-70$.

13 Kirson LE, Wilson ME. Atrial fibrillation associated with intravenous fluorescein. Anesth Analg 1987; 66: 283.

14 Butner RW, McPherson AR. Adverse reactions in intravenous fluorescein angiography. Ann Ophthalmol 1983; 15: $1084-6$.

15 Karhunen U, Raitta C, Kala R. Adverse reactions to fluorescein angiography. Acta Ophthalmol $(K b h)$ 1986; 64: $282-6$.

16 Yannuzzi LA, Roherer KT, Tindel LG, et al. Fluorescein angiography complication survey. Ophthalmology 1986; 93: 611-7.

17 Elman MJ, Fine SL, Sorenson J, et al. Skin necrosis following fluorescein extravasation. A survey of the macula society. Retina 1987; 7: 89-93.

18 Arroyave CM, Wolbers R, Ellis PP. Plasma complement and histamine changes after intravenous administration of sodium fluorescein. Am $\mathcal{f}$ Ophthalmol 1979; 87: 474-9.

19 Eustace P, Travers S, Mooney D. Oral Fluorescein angiography in disc oedema. Bull Soc Belge Ophtalmol 1984; 211 : $35-41$

20 Jacob JSH, Rosen ES, Young E. Report on the presence of a toxic substance, dimethyl formamide, in sodium fluorescein used for fluorescein angiography. Brf Ophthalmol 1982; 66: $567-8$.

21 Buckley $\mathrm{H}$. Fluorescein injection. DHSS communication. Reference no. 2-10/81. 23 June 1982.

22 Morgan KS, Franklin RM. Oral fluorescein angioscopy in aphakic children. $\mathcal{F}$ Pediatr Ophthalmol Strabismus 1984; 21 : 33-6.

23 Ghose $S$, Nayak BK. Role of oral fluorescein in the diagnosis of early papilloedema in children. Br $\mathcal{F}$ Ophthalmol 1987; 71: 910-5.

24 Bynke HG. Differentiation of papilloedema from pseudopapilloedema by fluorescein ophthalmoscopy. Acta Ophthalmol $(K b h)$ 1970; 48: 752-8. 